\title{
LES MALADIES DES POISSONS
}

\author{
par le Dr. P. W. SCḦ̈PERCLAUS
}

\section{AVANT-PROPOS}

Dans la $3^{\mathrm{e}}$ édition de son livre universellement connu "Les maladies des Poissons ", le Dr. P. W. Sch Äperclaus étudie de façon approfondie la plupart des maladies s'attaquant aux poissons et les moyens de les combattre.

Les 643 pages consacrées par le Dr. Sch Äperclaus à cette question, comprennent une documentation fort détaillée et intéressante. Il est peu probable que son ouvrage sera traduit en français, mais nous pensons que le résumé que nous en donnons ci-après pourra néanmoins préparer les pisciculteurs et les naturalistes s'occupant d'ichtyologie, à une meilleure connaissance des maladies dont les poissons peuvent être atteints.

Bien des points faisant l'objet de développements parfois importants dans l'ouvrage du Dr. Sch ̈̈perclaus, tels les malformations, les kystes, les tumeurs et certaines maladies qui affectent plus particulièrement les poissons vivant en eau libre, n'ont pas été abordés dans notre résumé, qui concerne plus particulièrement les maladies observées sur les poissons de pisciculture et d'aquarium.

La bibliographie contenue dans le live du Dr. Schäperclaus, qui occupe 29 pages et comprend plus de 600 articles, serait également fort intéressante à parcourir et atteste le développement des recherches sur les maladies des poissons dans certains pays.

L'ouvrage du Dr. Schäperclaus a été édité par l'Akademie Verlag G. m. b. H. ’̀ Berlin, Leipziger St. 3-4.

B. Dussart et Ch. Mercier.

\section{TRAITEMENTS EXTERNES ET INTERNES}

\section{A. LUTTE CONTRE LES PARASITES DE LA PEAU ET DES BRANCHIES}

Pour lutter contre les parasites de la peau et des branchies, on peut utiliser des bains soit de courte durée, soit de longue durée. 


\section{A. - Bains de courte durée.}

\section{Bains de sel de cuisine :}

a) Bains forts pour les gros poissons :

$25 \mathrm{gr}$. de sel dans un litre d'eau (solution à 2,5\%).

Durée du bain : 10 à 15 minutes.

b) Bains faibles pour les jeunes poissons et pour les poissons d'aquarium :

10 à 15 gr. dans un litre d'eau (solution de 1 à $1,5 \%$ ).

Durée du bain : 20 minutes.

Les bacs en zinc ne doivent pas être employés pour ces bains, car il se formerait du chlorure de zinc, nocif.

Le tableau ci-dessous fournit quelques données concernant l'action de ces bains de sel de cuisine, sur le parasite "Costia ", à la température de $19^{\circ}$ et sur des Carpes de $15 \mathrm{~cm}$. de longueur.

\begin{tabular}{|c|c|c|c|}
\hline & \multicolumn{3}{|c|}{ Concentration des Bains } \\
\hline Effet du Bain. & $1 \% \mathrm{Na} \mathrm{Cl}$ & $1,75 \% \mathrm{Na} \mathrm{Cl}$ & $2,5 \% \mathrm{Na} \mathrm{Cl}$. \\
\hline $\begin{array}{c}\text { Encore des "Costia" } \\
\text { vivantes. }\end{array}$ & $\begin{array}{l}\text { après } 10 \mathrm{mn} . \\
\text { de bain. }\end{array}$ & $\begin{array}{l}\text { après } 3 \mathrm{mn} . \\
\text { de bain. }\end{array}$ & $\begin{array}{l}\text { après } 20 \text { à } 25 \\
\text { secondes de bain. }\end{array}$ \\
\hline $\begin{array}{c}\text { Plus aucune " Costia " } \\
\text { vivante }\end{array}$ & après $15 \mathrm{mn}$. & après $4 \mathrm{mn}$. & après $1 / 2 \mathrm{mn}$. \\
\hline $\begin{array}{l}\text { Moment où le poisson } \\
\text { commence à se mettre } \\
\text { sur le flanc. }\end{array}$ & & après $3 \mathrm{mn}$. & après $1 / 2 \mathrm{mn}$. \\
\hline
\end{tabular}
sont tués :

- Avec des bains de sel de cuisine à $1 \%$ les parasites suivants

Costia en 32 minutes;

Chilodonella en 2 minutes;

Cyclochaeta en 1 minute.

- Des bains de sel de cuisine à $3 \%$ (30 gr. de $\mathrm{Na} \mathrm{Cl}$ dans un litre d'eau) doivent détruire la "Mousse "chez les poissons.

- On recommande ces bains de sel de cuisine $(0,75$ à $1,10 \%)$ pour détruire les Lernaea. En les utilisant plusieurs fois au cours de deux 
à trois semaines, les larves doivent être complètement tuées. Par contre, tous les poissons résistent bien lorsque, sortant d'une solution à $0,7 \%$ on les habitue peu à peu à une dose de sel plus forte : de 1,4 à 1,5\%. de mucus.

- Les bains de sel de cuisine, enfin, favorisent la production

\section{$2^{\circ}$ Bain de sulfate de cuivre :}

Des solutions à 1 gr. pour 50 litres sont supportées pendant des jours par des poissons particulièrement résistants, mais il y a toujours quelques individus sensibles qui meurent.

Pour la lutte contre les parasites visibles de la peau et des branchies on recommande :

Bain à $1 \mathrm{gr}$. de sulfate de cuivre dans 10 litres d'eau.

Durée du bain : 10 à 30 minutes.

- Les Costia sont pratiquement tués au bout de 10 minutes.

- Les Gyrodactylus supportent 30 minutes de bain.

Dans ce bain les poissons deviennent passablement agités, leur peau devient plus ou moins légèrement blanchâtre.

$3^{\circ}$ Bain de sel ammoniac ou de chlorure d'ammonium :

Rarement utilisé parce qu'il n'offre aucun avantage particulier, il était autrefois recommandé contre les Gyrodactylus.

Bain à 10 à 25 gr. de sel ammoniac $\left(\mathrm{NH}_{4} \mathrm{Cl}\right)$ dans un litre d'eau (solution à 1,5 à $2,5 \%$ ).

Durée du bain : 10 à 15 minutes.

\section{Bain d'ammoniaque (alcali) :}

a) Bains forts : $1 \mathrm{~cm}^{3}$ par litre d'eau.

b) Bains faibles : $0,5 \mathrm{~cm}^{3}$ par litre d'eau.

Durée du bain : 4 à 7 minutes.

Ils sont employés contre les parasites de la peau, mais peu recommandables.

\section{5o Bain d'eau oxygénéè :}

Ils ne présentent aucun arantage particulier.

Utiliser un bain à $17 \mathrm{~cm}^{3}$ d'une solution à $3 \%$ dans un litre d'eau. Durée du bain : 5 minutes.

\section{$6^{\circ}$ Bain de permanganate de potassium :}

à 1 gr. dans 100 litres d'eau.

Manière de procéder : $1 \mathrm{gr}$. de cristaux de permanganate est dissous dans $100 \mathrm{~cm}^{3}$ d'eau. De cette solution de base, $1 \mathrm{~cm}^{3}$ est dilué dans un litre d'eau. 
d'eau.

On peut utiliser aussi : $1 \mathrm{gr}$. de pesmanganate dans 10 litres

Durée du bain : 5 à 10 minutes.

Ce bain est utilisé contre les Argulus.

Pour détruire Costia, Chilodonella et Cyclochaeta, on recommande un bain de 10 minutes, mais quelques jours après le bain on trouve encore quelques parasites.

Pour lutter contre la "Mousse "(Saprolegnia) il est recommandé :

$1^{0}$ D'enlever la plus grande partie des champignons en frottant la peau avec une éponge ou un tampon de coton hydrophile, trempés dans une solution de permanganate à $1 / 1.000$ ( $1 \mathrm{gr}$. dans un litre d'eau).

$2^{\circ}$ De baigner ensuite les poissons dans une solution à $1 \mathrm{gr}$. pour 100 litres d'eau, pendant 90 minutes.

70 Bain de chlore :

Ce bain doit être appliqué avec du chlorure de chaux de telle manière que dans l'eau on trouve $1 \mathrm{mgr}$. de chlore libre. (Lernaea).

On utilise ce bain contre les Copépodes parasites de la peau

\section{$8^{\circ}$ Bain de chlorure de chaux:}

Mettre 1,5 à 2 gr. de chlorure de chaux dans 1.000 litres d'eau. Durée du bain : 1 heure à 1 h. $1 / 4$.

Les Carpes supportent un bain à $16 \mathrm{mgr}$. de chlore par litre, et à $16^{\circ} \mathrm{C}$ pendant 45 heures; ou un bain à $5 \mathrm{mgr}$. de chlore par litre, à 4 ou $6^{\circ} \mathrm{C}$ pendant 21 heures.

\section{9o Bain de "Caporite "} d'eau.

On utilisera un bain de 0,5 à 1 gr. de "Caporite " pour 1.000 litres

Durée du bain : 10 à 30 minutes.

La Caporite (ou Losantin) est de l'hypochlorite de calcium pur ajouté à du sel de cuisine [Ca $\left.(\mathrm{OCl})_{2}+\mathrm{Na} \mathrm{Cl}\right]$. Elle contient 70 à $80 \%$ de chlore actif et elle est trois à quatre fois plus active que le chlorure de chaux.

Un inconvénient de ces bains de chlore (en particulier pour la lutte contre les sangsues) est la persistance du chlore, élément. nocif, dans le milieu et la nécessité donc, de changer fréquemment par la suite le liquide dans lequel vivent les poissons.

\section{$10^{\circ}$ Bain d'acide salicylique :}

Dans une eau à $20^{\circ}$, faire une solution concentrée d'acide salicylique en poudre et mettre 1 litre de cette solution dans 20 litres d'eau.

Ce bain est utilisé contre Gyrodactylus, on recommande alors une solution à $0,25 \%$ d'acide salicylique. 
Durée du bain : 30 minutes.

Ce bain ne présente aucun avantage particulier, il n'est que peu employé aujourd'hui.

\section{Bain de formol :}

Recommandé tout particulièrement (ainsi que le bain de sel de cuisine) pour la lutte contre Gyrodactylus et Dactylogyrus. Il agit également fort bien contre les Costia.

La dose à prescrire est de $35 \mathrm{~cm}^{3}$ de formol pour 100 litres d'eau en moyenne. d'eau.

Pour les bains forts on peut aller jusqu'à $100 \mathrm{~cm}^{3}$ pour 100 litres

Durée du bain : 15 minutes. d'eau.

Pour les bains faibles, la dose va de 20 à $50 \mathrm{~cm}^{3}$ pour 100 litres

Durée du bain : 30 à 45 minutes.

120 Bain de zéphirol :

C'est un chlorure d'Alkyl-diméthyl-Benzyl-Ammonium, à utiliser en dilutions de $1 / 2.000$ à $1 / 4.000$.

Durée du bain : 20 à 30 minutes. spécial.

Ce bain agit comme le formol, mais ne présente aucun avantage

\section{B. Bains de longue durée.}

Ces bains sont employés, par exemple, pour lutter contre Ichthyophtirius, et presque uniquement pour les poissons d'aquarium.

Les produits les plus souvent employés sont la Trypaflavine, le Rivanol, l'Atebrine et la Quinine.

\section{1" Bain de quinine :}

On utilisera des sels de Quinine.

Il faut que toute la quinine soit réellement dissoute avant d'être mise dans les bassins.

Bain fort : 1 gr. de chlorure de quinine ou de sulfate de quinine dans 50 litres d'eau.

Bain moyen : 1 gr. dans 75 litres d'eau.

Bain faible : 1 gr. dans 100 litres d'eau.

Les bains forts ne doivent être employés qu'avec des poissons résistants ou lorsque les bancs et aquariums renferment beaucoup de plantes et possèdent un fond sain. En effet, une partie de la quinine est absorbée ou fixée par les plantes et par le sol. 
Les bains de longue durée peuvent détruire non seulement les Ichthyophtirius, mais également les autres protozoaires parasites de la peau : Costia, Chilodonella, Cyclochaeta.

\begin{tabular}{|c|c|c|c|c|}
\hline $\begin{array}{l}\text { Concentration } \\
\text { de la Solution } \\
\text { de Chlorure de Quinine }\end{array}$ & & Costia & Chilodonella & Cyclochaeta \\
\hline 1g./10 l. d'eau. & $\begin{array}{l}\text { tués après } \\
4 \text { à } 8 \text { heures. }\end{array}$ & & $\begin{array}{l}\text { tués après } \\
4 \text { à } 6 \text { heures. }\end{array}$ & \\
\hline 1g./50 l. d'eau. & $\begin{array}{c}\text { tués après } \\
9 \text { à } 18 \text { heures }\end{array}$ & $\begin{array}{c}\text { tués après } \\
24 \text { à } 26 \text { heures }\end{array}$ & $\begin{array}{c}\text { après } \\
18 \text { heures. }\end{array}$ & $\begin{array}{l}\text { après } \\
6 \text { heures. }\end{array}$ \\
\hline 1g./100 l. d'eau. & $\begin{array}{c}\text { tués après } \\
24 \text { à } 48 \text { heures }\end{array}$ & & & $\begin{array}{l}\text { vivants après } \\
2 \mathrm{j} \text {. et } 5 \mathrm{~h} \text {. }\end{array}$ \\
\hline
\end{tabular}

Les Ichthyophthirius libres sont tués après les temps suivants, à $20^{\circ} \mathrm{C}$ et selon la concentration du bain :

1 gr. de sulfate de quinine par litre d'eau : 20 minutes;

1 gr. de sulfate de quinine dans 10 litres d'eau : 45 minutes; ron ;

1 gr. de sulfate de quinine dans 50 litres d'eau : 3 heures envienviron.

$1 \mathrm{gr}$. de sulfate de quinine dans 100 litres d'eau : 7 heures

2o Bain de Trypaflavine, de Rivanol et d'Atebrine :

Ces corps sont des dérivés de matières colorantes.

Dissoudre $1 \mathrm{gr}$. de Trypaflavine ou de Rivanol dans 100 litres d'eau. Les poissons supportent mieux ces bains que ceux de quinine; ils ne causent pas de grands dommages aux plantes d'aquarium.

Leurs autres avantages sont : leur prix modéré et la possibilité de reconnaître, à la coloration de l'eau, la juste concentration.

Les Chilodonella cyprini sont tués dans des solutions à $1 \mathrm{gr}$. pour 100 litres d'eau en 2 heures, ou plus exactement entre 2 et 10 heures. Mais les champignons sur les œufs des poissons ne sont pas détruits par la Trypaflavine ni le Rivanol.

De ces trois dérivés de l'Acridine, on recommandera surtout l'utilisation de la Trypaflavine contre Ichthyophthirius.

$3^{\circ}$ Bain d'argent :

Pour lutter chez les poissons contre les champignons de la peau 
et les inflammations bactériennes de la peau et des nageoires, on peut utiliser un bain d'argent.

Ce bain est relativement mal supporté par les poissons et assez bien par les parasites. On doute qu'il soit possible de détruire les bactéries de la peau ou des nageoires de cette façon.

Le " Collargol " (70\% d'argent colloïdal) peut être utilisé. Un bain de Collargol en contient 1 gr. dans 500 litres d'eau.

Le bain de "Micropur" (employé dans le procédé Katadyn pour la désinfection des eaux potables) est utilisé à raison de $1 \mathrm{gr}$. dans 100 litres d'eau. Pour les poissons sensibles, 1 gr. de Micropur sera dilué dans 150 litres d'eau.

\section{$4^{\circ}$ Bain de bleu de méthyléne :}

Bain à $3 \mathrm{~cm}^{3}$ d'une solution aqueuse à $1 \%$ de bleu de Méthylène $\mathrm{B}$ dans 10 litre d'eau.

Il est très bien supporté, même par les petits poissons, mais les Chilodonella ne meurent qu'après plusieurs jours : Gyrodactylus vit encore après cinq jours.

Ce bain ne convient pas pour la lutte contre Ichthyophtirius, car il est trop long; en outre, la peau des poissons est fortement teintée dans ce bain. Il n'est donc pas à recommander.

\section{$5^{\circ}$ Bain de chloramine :}

Utilisé pour la destruction des Planaires (contre lesquelles on recommande aussi les bains à 12 gr. de Nitrate d'ammonium pour 10 litres d'eau) et celle des hydres d'eau douce (contre lesquelles on pourra employer des bains à 1 gr. de Nitrate d'ammonium dans 10 litres d'eau).

La Chloramine est le sel de sodium d'un acide organique contenant du chlore, l'acide " $p$-Toluol-chloramid-sulfonique".

Bain : 1 gr. dans 15 litres d'eau.

Durée : de 2 à 4 heures.

ou 1 gr. dans 100 litres d'eau pendant plusieurs jours.

L'action sur les parasites est en général très bonne.

Costia : destruction en trois heures.

Gyrodactylus : destruction en quelques heures également.

Inconvénient : La concentration diminue rapidement, d'où nécessilé d'ajouter souvent de la Chloramine.

$6^{\circ}$ Bain de sulfamides : Globucide, Méthylsulfadiazine, Albucide.

Ces produits sont difficilement solubles.

On utilisera une solution à 2 gr. de sel sodique de la globucide ou de l'Albucide par litre d'eau (qu'il est possible d'acheter en ampoules contenant 2 gr. dissous dans $10 \mathrm{~cm}^{3}$ ).

Ces bains sont chers et ne sont nullement supérieurs à ceux de Trypaflavine toute seule. 
$7^{\circ}$ Bain de D. D. T. :

Employé pour la destruction d'Ergasilus, Achteres, Lernea, Argulus, etc. à raison de $1 / 50.000 .000$ à $1 / 100.000 .000$ de D. D. T. dissous dans l'eau.

Avec des bains concentrés à 20 gr. de Gésarol par litre d'eau, pendant 30 minutes, on a obtenu de bons résultats contre Ergasilus.

$8^{\circ}$ Bain de " chaleur :

Dans les aquariums, une simple élévation de la température jusqu'à $32^{\circ}$ pendant 5 jours et une lente baisse pendant 8 jours suffisent à faire disparaître les parasites (surtout Costia).

\section{Bains par immersion.}

Ce sont des bains de courte durée, quelques secondes seulement.

\section{Bain de Lysol :}

Il agit en peu de temps contre les Argulus.

Le Lysol est une solution de savon de Crésol qui contient $50 \%$ de Crésol brut (ortho-Meta-et Paracrésol) et $50 \%$ de savon de Potassium.

a) bain : solution à $0,2 \%$ ( 1 cc. de Lysol pour 5 litres d'eau) dans des baquets de bois.

b) On immerge les panniers contenant les poissons pendant 5 à 15 secondes.

c) On rince les poissons dans de l'eau fraîche.

d) Puis ils sont mis sur les tables de tri pour écarter les parasites qui pourraient rester (Argulus et sangsues).

e) Mettre de la chaux vive dans les baquets ayant servi au bain.

Après le bain, on note chez certains poissons quelques mouvements spasmodiques, mais tout rentrera dans l'ordre au bout de quelques minutes.

Les Costia et les Cyclochaeta restent en vie après un bain de 45 secondes.

Les Chilodonella meurent en 5 secondes.

Les Gyrodactylus vivent encore après 30 secondes, mais faiblement.

Les bains de Lysol sont efficaces dans la lutte contre les parasites microscopiques de la peau et des branchies, mais naturellement on ne doit pas les recommander pour la lutte contre les troubles contagieux de la peau car le formol, le sel de cuisine et la Trypaflavine sont, dans ces cas, de meilleurs remèdes.

$2^{\circ}$ Bain de chaux vive :

Il est employé dans la lutte contre les parasites visibles, à raison de 2 gr. de chaux dans un litre d'eau. 
Durée du bain : 5 secondes.

On procèdera comme pour le bain de Lysol.

Ce bain est excellent contre les sangsues parasites.

\section{$3^{\circ}$ Bain de permanganate de Potassium :}

Très utilisé, en solution à 1 gr. de permanganate par litre d'eau, contre les Argulus.

Durée du bain : 30 à 45 secondes.

Les poux n'abandonnent le poisson qu'après le bain (comme dans le bain de Lysol), souvent après 9, voir 24 heures.

Costia, Chilodonella et Cyclochaeta sont tués par une immersion de 45 secondes dans une solution à $1 / 1.000^{\mathrm{e}}$.

\section{Traitement par poudre.}

Pour lutter contre les Argulus on saupoudre les poissons de talc ou de fleur de soufre. Ces poudres sèches, non solubles dans l'eau, n'endommagent pas le poisson mais obligent les parasites à abandonner l'hôte dès que les poissons sont remis à l'eau.

Contre les Ergasilus, les Lernae, les Argulus, on recommande de saupoudrer la peau et les branchies des poissons avec du gérasol; les branchies ne sont pas endommagées si l'on remet les poissons dans un fort courant d'eau aussitôt après le traitement.

\section{B. TRAITEMENT DES MALADIES INTERNES}

\section{$1^{\circ}$ Par ingestion de médicaments et de bactériophages.}

\section{a) Les tumeurs de la Thyroïde.}

Pour ces tumeurs, ajouter à la nourriture une solution d'iode dans l'iodure de potassium (liquide de Lugol) dans la proportion de un litre pour 2.500 litres (une partie d'iode, une partie d'iodure de potassium, pour cent parties d'eau).

\section{b) Maladies dues à l'Octomitus.}

On recommande, en Amérique, contre cette maladie, un mélange d'apport de $0,2 \%$ de Calomel (Chlorure mercurique) pendant deux jours ;

$\mathrm{Ou}$ de $0,2 \%$ de Carbarsone (acide para-carbamido-phénylarsenique) $\left(\mathrm{H}_{2} \mathrm{O}_{3}\right.$ As. $\mathrm{C}_{6} \mathrm{H}_{4}$. $\left.\mathrm{NHCONH}_{2}\right)$ pour quatre jours.

\section{c) Ingestion de Phagocytes en cas de maladies infectieuses.}

Il est possible d'obtenir des bactériophages capables de détruire Pseudomonas punctata; leur grandeur est d'environ $50 \mathrm{~m} \mu$ et ils ne peuvent se multiplier qu'avec la "collaboration " de bactéries vivantes. 
Ces bactériophages pénètrent dans les bactéries, s'y multiplient très vite, et au bout de quelques minutes la bactérie peut être dissoute. Déjà, après une première addition de boue contenant des phagocytes et provenant d'un bassin ou d'un étang dans lequel se trouvaient des Carpes atteintes d'hydropisie, on a réussi à obtenir un net éclaircissement des bouillons de culture de Pseudomonas punctata. Comme on a réussi à élever en grand, par addition de Phénol, des Phagocytes sans bactéries vivantes, rien ne s'oppose à une ingestion massive de bactériophages rétruisant Pseudomonas punctata.

Chez les Truites, le nourrissage avec des bactériophages peut être pris en considération en cas de furonculose.

\section{$2^{\circ}$ Traitements particuliers.}

S'il s'agit de maladies provenant de circonstances extérieures ou d'erreurs de nourrissage, il est possible de remédier à ces inconvénients. La guérison peut être laissée au soin de la nature grâce à un jeûne de une à deux semaines par exemple.

La méthode de guérison naturelle peut s'employer avec les poissons comestibles; après les bains, ils doivent être mis aussitôt dans de grands étangs riches en nourriture, avec une eau convenable.

Dans maintes maladies, comme celle due à l'Ichthyophthonus, il suffit d'accorder aux poissons de meilleures conditions de vie, par exemple de leur éviter le manque d'oxygène et les écarts de température. Deux Inéthodes peuvent être employées ; le premier procédé emploie la méthode des "changements de place ": les poissons atteints par Ickínyophtirius sont placés toutes les douze heures dans un bassin nouveau, libre de parasites.

Dans la seconde, dite méthode du "lavage ", les poissons atteints sont placés dans des paniers suspendus dans un fort courant d'eau, de telle sorte que les parasites qui tombent sont emportés par le courant.

30 Prophylaxie et guérison par vaccination, ainsi que par injection de remèdes chimio-thérapeutiques et autres.

L'avantage de l'immunisation active provient de la durée de la protection, son désavantage réside dans le long délai que demande la formation des substances protectrices.

L'immunisation passive a le privilège de n'inoculer aucune maladie et d'agir immédiatement. On l'emploie donc avant tout pour la guérison des maladies déjà bien déclarées. Par contre, cette immunisation passive ne dure pas longtemps et elle est relativement coûteuse.

Certains chercheurs se sont servis de l'immunisation active chez les poissons, mais en pratique elle n'est employée que pour des cas bien définis, par exemple dans de petits élevages. Elle est sans succès dans les élevages importants. 
Les méthodes chimio-thérapeutiques sont plus efficaces, elles comprennent :

$1^{\circ}$ Les remèdes antibactériens, bactéricides, comme la Trypaflavine, le Rivanol.

$2^{\circ}$ Les remèdes bactériostatiques, empêchant la croissance et la multiplication des bactéries :

a) Remèdes synthétiques, par exemple les Sulfamides (p. aminobenzol-sulfamide $\mathrm{H}_{2} \mathrm{~N}<\longrightarrow \mathrm{SO}_{2} \mathrm{NH}_{2}$ (Sulfa - ) ; le sulfa-thiazol et la sulfa-pyrimidine sont particulièrement intéressants.

b) Antibiotiques.

$3^{\circ}$ Les Excitants : tels que les colloïdes métalliques, le Soufre, les Albuminoïdes, l'essence de térébenthine.

Avec certains sulfamides on a obtenu quelques succès dans le traitement de l'hydropisie infectieuse en petits élevages. On peut employer traitement de l'hydropisie infectieuse en petits élevages. On peut employer :

a) Le Prontosil (4-Sulfonamid 2', 4'-diamino-azobenzol) 1 gr. pour $1 \mathrm{~kg}$. de poisson.

b) Globucide ( $\mathrm{p}$ - Aminobenzolsulfonamido - aethyl - thiodiazol) $1,6 \mathrm{gr}$. pour $1 \mathrm{~kg}$. de poissons.

c) Pyralcide (1 gr. pour $1 \mathrm{~kg}$. de poisson). Le traitement dans un grand élevage est trop coûteux pour être considéré. 